\title{
An Alternative to Waves and Wave-Packets
}

\author{
Bill Gaede
}

\begin{abstract}
Classical and Quantum Mechanics invoke waves, wave-packets and particles to provide physical interpretations to electromagnetic phenomena. However, these illustrations are neither supposed to be taken literally nor are they advertised as genuinely depicting the real world. Their sole purpose is to represent invisible interactions classically in order to provide a measure of physicality to the mathematical descriptions. It is thus that certain experiments are simulated with extended waves and others with discrete wave-packets. Duality raises questions as to the ad hoc nature of the representation and to the usefulness of abstractions as didactic and explanatory tools. Here we suggest that light has been misdiagnosed. We propose an alternative model that provides a faithful physical interpretation to the fundamental properties of light. We replace the electromagnetic wave with a two-strand rope and simulate the wave-packet as a torsion propagating from atom to atom along this twined entity. The fundamental assumption is that all atoms in the Universe are interconnected. The rope hypothesis justifies and simulates basic features and behaviors of light such as straightness, speed, and orthogonality, and merges light with gravity into a single mechanism.
\end{abstract}

Index Terms-Light, gravity, rope hypothesis, electromagnetic wave, particle, $c=f \lambda$, slit experiment, EPR, polarization, GUT

\section{INTRODUCTION}

This paper presents an alternative physical interpretation for light that is consistent with equations, observations and experiments. Therefore, a quick review of the evolution of ideas about light's structural nature is in order.

For most of human history, enthusiasts have regarded light to consist of discrete particles. It wasn't until about 400 years ago that the wave model began its gradual ascent and grew to be so popular that theorists practically abandoned the particle hypothesis by the $19^{\text {th }}$ Century. In the first quarter of the $20^{\text {th }}$ Century, researchers merged both models into a mathematical duality known as 'wave-packet'. The Complementarity Principle still in vogue today resolved that light has both particle and wave characteristics and that the nature of the experiment determines which of these aspects light will exhibit. ${ }^{1}$ Theorists had by then lost hope of discovering what light 'is' and turned their attention to describing how it behaves during an experiment.

Today, a little over 80 years since the seminal $5^{\text {th }}$ Solvay Conference, we present a new physical model that stands in parallel with the particle, the wave and the wave-packet. We begin by assuming that a physical medium underlies the phenomenon known as 'light' and that this entity takes on the configuration of a rope. The rope hypothesis provides a

Manuscript received May 24, 2011; revised August 25, 2011.

Bill Gaede is with ViNi, Frankfurt, Germany (email: viligaede@yahoo.com). wholly different perspective to the workings of the Universe.

\section{A PhysiCAl MOdel OF Light?}

The mere suggestion that light could be mediated by a physical entity may sound strange and anachronistic to many a reviewer. We have not pondered such questions since the early $20^{\text {th }}$ Century when researchers and theorists finally abandoned classical models. And yet again, we are able to interrupt light with our hands and generate shadows. In unusual circumstances, it is sometimes necessary to exhume the body and perform a new autopsy in order to reassure all sides of the debate that all the Ts have been crossed. It behooves us as scientists to reconsider the decision to regard light as an abstraction, especially when a new proposal is on the table. A physicist seeks more than just mathematical symbolism and wishes to understand how Mother Nature actually performs her invisible tricks. This noble quest should not be summarily taken away in one fell swoop through censorship.

Einstein, for one, tacitly invoked a physical model to explain the photoelectric effect and, although his was primarily a mathematical theory, he did insinuate and explain that discrete bundles of light dislodged discrete electrons from the atoms comprising the polished metal. ${ }^{2} \mathrm{We}$ may also argue that the particles of the Standard Model of Quantum are not really physical, yet all explanations that invoke particles by default treat them as such.

\section{The EM RoPe IS QuiTe UnLIKE A 'WAVE'}

At face value, the rope doesn't appear to be much different than the electromagnetic (EM) wave of Classical Mechanics. The EM wave would seem to be but a cross-section of a rope.

However, a couple of distinctions are in order. Whereas the Fresnel - Maxwell classical wave is an abstract mathematical artifact, the rope is presented as a real physical medium. The EM rope is comprised of two threads twined around each other like strands of DNA (Fig. 1).

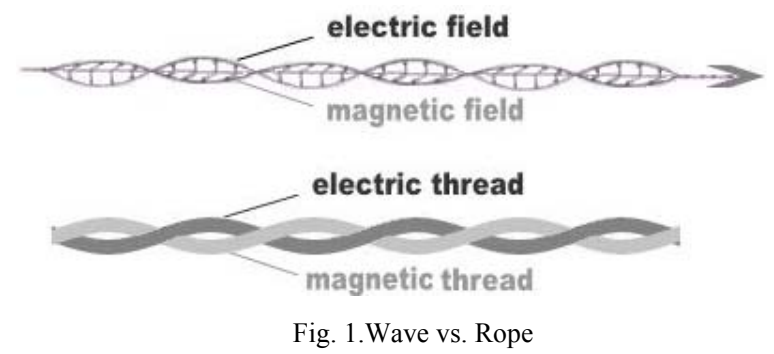

The plane transverse wave is an ad hoc, two-dimensional tool that few scientists, if any, argue exists out there. Wave theory holds that an atom emits the classical wave outwards. 
Quantum Mechanics adds that the wave condenses into a particle upon contact with another atom. Baierlein synthesizes our understanding of the behavior of light in his maxim: "Light travels as a wave and departs and arrives as a particle." 3

In contrast, we postulate that the EM rope extends from one atom to all others, thus binding any two atoms of the real world. Does the classical plane transverse wave perchance extend from an atom in the Andromeda Galaxy all the way to Earth (Fig. 2)? If not, where does it start? Where does it end? These questions would seem to have a simpler answer if we replace the abstract EM wave with the rope model of light.

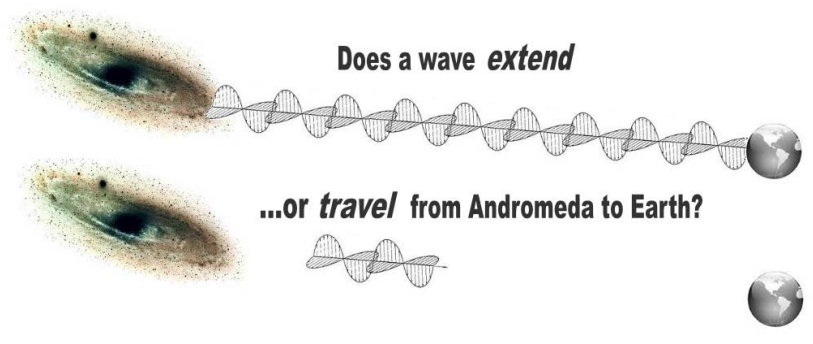

Fig. 2.Does an EM wave extend or travel?

From a dynamic perspective, the EM wave propagates in a single direction. Waves, particles and wave-packets are all one-way mechanisms. The EM rope, instead, is a structural entity that binds any two atoms and torques its twined threads in situ. 3D torsion waves travel simultaneously in opposite directions along this physical mediator. We suggest that these torsion waves have been mistaken for wave-packets.

\section{How the Rope Model MeAsures UP}

As a bare minimum, an impartial juror should check that a physical candidate be able to simulate the most fundamental properties and behaviors of light. Consequently, we list ten of the most salient structural and dynamical features that any candidate should exhibit before it can be considered as a serious contender for the job.

\section{A. Frequency, wavelength and amplitude}

Whereas Wave Theory treats frequency, wavelength and amplitude as dynamic parameters, the rope model proposes them as architectural attributes. An EM rope has structural frequency in the number of links, and wavelength, in the length of each link (Fig. 3). The amplitude is given by the height of the link.

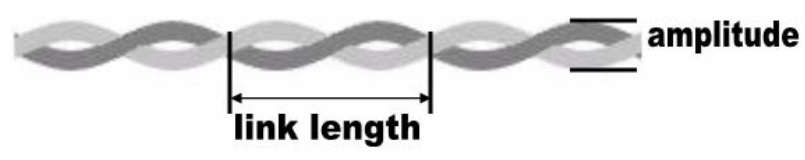

Fig. 3.Linklength and amplitude

\section{B. Speed}

The EM wave has been calculated to travel at 300,000 $\mathrm{km} / \mathrm{sec}$, which is widely regarded to be the speed limit of the Universe. Orthodoxy has never explained why light travels so fast. It is pertinent to note that longitudinal P-waves have been timed to travel almost twice as fast as transverse shear (S) waves during earthquakes. ${ }^{4}$ If we were to extrapolate the analogy to longitudinal sound and transverse light waves, we would hear the sound of thunder before seeing the lightning flash.

Both longitudinal and transverse waves are no match for torsion waves. A simple experiment shows just how fast they are: it is practically impossible to film the speed of signal transmission along a taut rope. By merely touching a clothespin at one end of a clothesline, the one at the other end moves 'instantly'.

It is also calculated that light travels around 220,000 $\mathrm{km} / \mathrm{sec}$ through glass. The classical wave fails to explain the mechanism or agent that accelerates light back to 300,000 $\mathrm{km} / \mathrm{sec}$ after the beam returns to atmosphere or vacuum. Certainly, the different colors generated by light refracting through a prism are attributed to a change in wavelength. ${ }^{5}$ It is, thus, by fixing frequency that theorists have concluded that the 'constant' $\mathrm{c}$ declines as light disperses through a prism.

Under the physical rope hypothesis, light travels at $300,000 \mathrm{~km} / \mathrm{sec}$ through any medium. Color is a function of link-length (Fig. 4) and the speed of the torsion along the rope remains the same irregardless of the material it extends across. What changes is the length of the links and, thus, the frequency (number of links per unit length) of light as the signal travels through different media (Fig. 5). Refractive Index (change in the speed of light) is a mathematical artifice that bears little relation to what is physically happening.

\section{C. $(c=f * \lambda)$}

The rope is the only physical configuration that explains why frequency is inversely proportional to wavelength. We can double the number of links on a 1-meter length of rope only at the expense of making the links shorter (Fig. 5). Maxwell's equation $\mathrm{c}=f * \lambda$ describes a rope. Wave Theory offers no physical interpretation for this observation. Under wave theory there is no physical impediment to increasing both frequency and wavelength.



Fig. 4.Color is a function of link-length

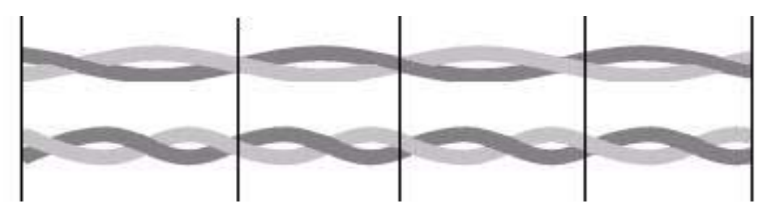

Fig. 5. Why $c=f * \lambda$

\section{Orthogonality, sinusoidality, bidirectionality and Straightness}

Faraday's Law states that an oscillating magnetic 'field' generates an electric field. ${ }^{6}$ Ampère/Maxwell's Law states that an electric 'field' induces a magnetic 'field'. ${ }^{78}$ Both run perpendicular to each other and for unexplained reasons 
oscillate around an imaginary axis.

The taut EM rope simulates all of these features. It has a magnetic strand running opposite to the electric fiber. The threads run perpendicular to each other in anti-parallel directions and justify why they oscillate around an imaginary axis (Fig. 6).

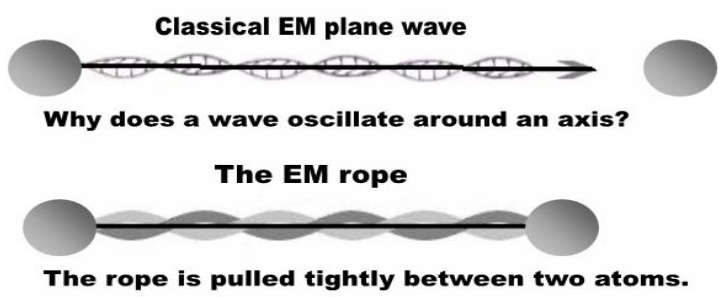

Fig. 6.Why does a plane wave oscillate around an axis? What compels it to travel rectilinearly?

A rope is also inherently wavy. The undulations are, in first instance, structural rather than dynamic. Of course, as the rope twirls, the torsion signals propagate simultaneously in diametrical directions and explain why we perceive them as sinusoidal waves.

Although as just discussed, at the very fine level a wave is known to oscillate, experimentalists usually deal with 'beams' or 'rays'. If a ray of light is comprised of particles, the particles themselves may not be said to 'be' straight. It is the ray that is straight. The particles can - if at all - 'travel' rectilinearly. Likewise, a series of peaks and valleys comprising an EM wave may not be said to 'be' straight. The wave-packet is alleged to 'travel' away from an atom, in which case it may at best do so rectilinearly (Fig. 6).

At first impression, this argument may strike as a trivial semantic issue. It is not. Again, does the classical EM wave begin in an atom and extend uninterruptedly to the next surface it touches or does the wave travel from one atom to the next like a sailboat floating from one shore to another? If it travels as a discrete entity, where does the wave start? Where does it end (Fig. 2)?

The issue of whether light is bent by warped space is also under siege and goes deeper than the superficial issue of language. The proponent may argue that a 'ray' or beam is bent. A series of discrete particles may at best be deflected (Fig. 7).

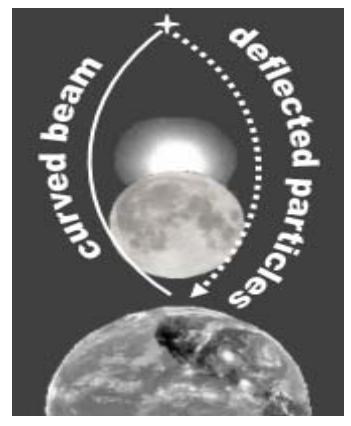

Fig. 7.Does warped space bend or deflect light?

However, the well-established Principle of Ray Reversibility, ${ }^{9} 10$ derived from Heron and Fermat's Principles, ${ }^{11}$ states that whether reflecting or refracting, a beam of light retraces its path. It is difficult to believe that a stream of discrete particles can continue to travel perfectly rectilinearly while the Earth moves at $30 \mathrm{~km} / \mathrm{sec}$ around the Sun. And if this micro-scenario is not persuasive enough, perhaps a macro one is. The U.S. and Russia routinely send laser signals to retro-reflectors that have been installed on the Moon in order to measure distances to our satellite accurately. ${ }^{12}$ The Moon is slightly over one light-second away and, therefore, the round trip for a photon would take a little over two seconds. A particle emitted from Earth would have trouble retracing its identical path after the Earth moved some $70 \mathrm{~km}$ from origin, especially if space is also warped (Fig. 8) and more so if we factor that "light 'travels' as a wave and departs and arrives as a particle." 3

The rope model avoids these predicaments since every atom comprising the Moon is bound to every atom comprising the Earth via twined EM threads (Fig. 9). A torsion has no chance of getting lost because it consists of the twisting of the elongated mediator itself.
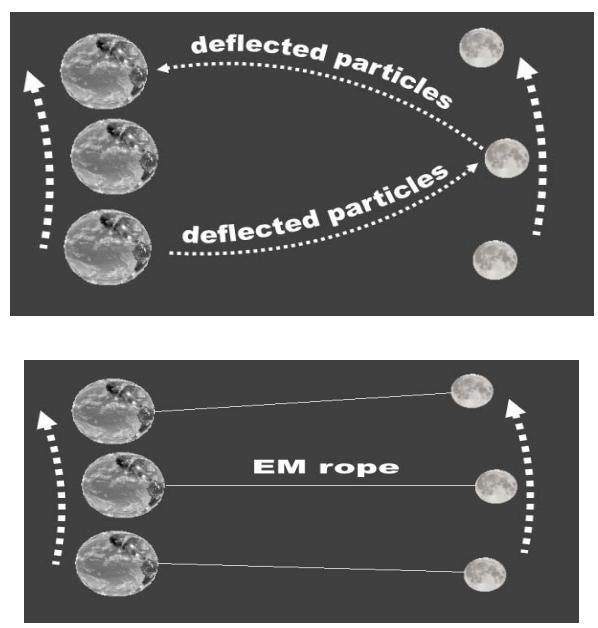

Figs. 8 and 9.The discrete particle and rope versions of the Lunar Ranging Experiment. (For simplicity, only one interconnecting rope is shown.) One-way entities such as particles, waves and wave-packets cannot justify the Principle of Ray Reversibility.. This optical phenomenon can only be justified by a two-way mechanism.

\section{E. Reflection and Refraction}

Newton postulated that light consists of corpuscles and, as a result, predicted that light should travel faster through glass than through air. Huygens' wave theory predicted the opposite. Unable to settle the matter at the time due to technological limitations, Huygens ended up winning the debate posthumously.

The rope is clearly more like the EM wave than it is like a discrete particle. Where the rope and the wave differ is in that the atoms comprising the mirror are already connected via twined EM threads to every atom comprising the test object as well as to the atoms comprising the eyes that see it (Fig. $10)$. Whether reflecting or refracting, every atom relays the specific torsion link-length to the next one.

\section{F. Diffraction}

Francesco Grimaldi appears to be the first observer to document that light indeed bends around corners. ${ }^{13}$ Thomas Young would later run a more formal experiment during which he measured the wavelength. ${ }^{14}$ His experiment and subsequent ones run by Augustine Fresnel to explain 
polarization would give the wave model an enormous boost in the eyes of their contemporaries. Maxwell and Hertz would put the final touches on the wave from both mathematical and experimental perspectives. The particle model was, thus, all but forgotten throughout most of the $19^{\text {th }}$ Century.
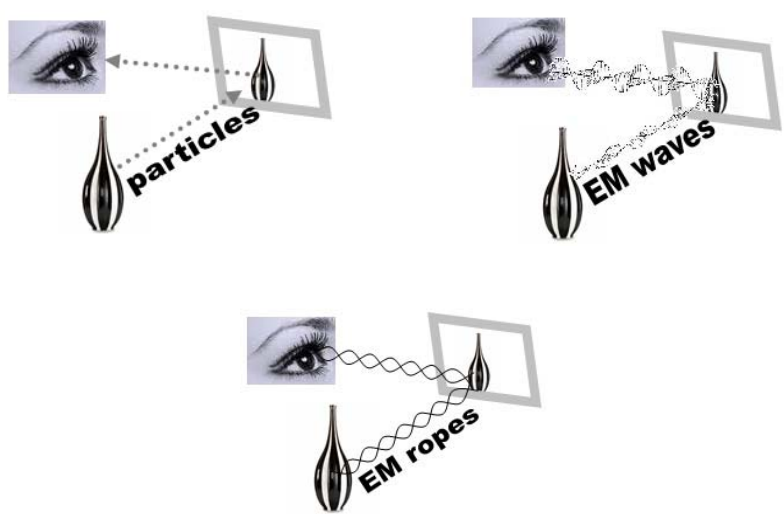

Fig. 10.Reflection: One-way particles and waves versus the interconnecting EM rope.

A rational physical interpretation of diffraction can succeed only by assuming that light is some sort of wave. For instance, several slit experiments have been performed by groups claiming to have handled individual electrons. ${ }^{1516}$ In order to prevail with their theories, however, these researchers tacitly invoked Bohr's admittedly debunked planetary model of the atom. ${ }^{17}$ In effect, the teams treated an electron as a discrete bead that orbits the nucleus. If we reject the planetary model of the atom, we must also reject claims based on such a model.

But even the one-way EM wave struggles to explain the slit experiment, a phenomenon that does not necessitate of slits to produce fringes and which can be easily performed with a laser pointer directed at a needle stuck to a cork. How does a one-way agent such as a plane transverse wave turn the corner inwards after passing the needle? Any one-way mediator should be expected to strike the edge of the needle and bounce outwards. And again, the question remains as to whether a wave is an entity that extends from one atom to another or travels as a discrete packet off to nowhere.

On the other hand, the rope hypothesis is at home explaining diffraction whether with a needle (Fig. 11) or with double slits (Fig. 12 A). Each atom from the source is connected via EM threads to each atom comprising the slit partition (or the edges of the needle) which are connected to each atom comprising the screen. When the light is turned on, the ropes torque at a higher frequency (links become shorter) and the atoms relay this signal from one to the other

The alleged bending of light by warped space is explained in like manner. The atoms comprising the Sun's corona relay EM torsion waves arriving from a star to the observer on Earth (Fig. 12 B).

\section{G. Spin}

Spin is a strange parameter. Most laymen imagine it as a ball rotating on its axis like a top. However, the 'static' language used to qualify spin - orientation, direction and pointing - as well as the numbers assigned to designate spin $1 / 2,-1,0$ - indicate that spin is a little more complex and quite unlike angular momentum.

The rope offers us a different perspective. Seen head on, we imagine seeing the ends of two strands. They either rotate clockwise (CW) or counterclockwise (CCW) (Fig. 13). A tiny observer traveling along the rope would nevertheless see the threads twine either $\mathrm{CW}$ or $\mathrm{CCW}$ even before the rope begins to rotate. The helical torsion of a rope, like the anti-parallel, hellical backbones of DNA molecules, is in principle a structural attribute.

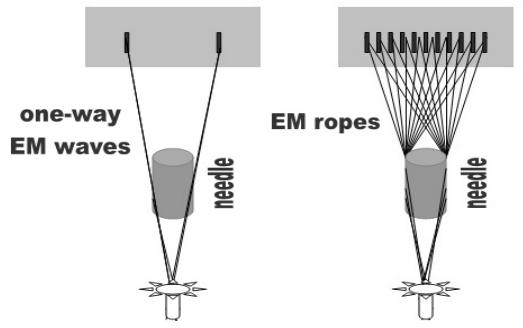

Fig. 11.Needle Experiment: one-way waves vs. interconnecting ropes.

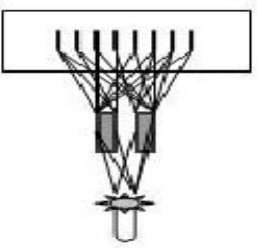

A

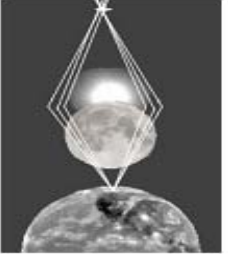

B
Fig. 12.A. Slit Experiment. B. Gravitational Lensing

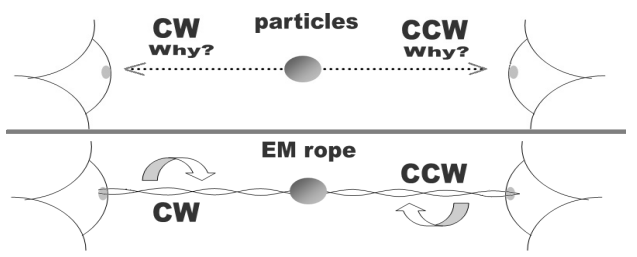

Fig. 13.EM Rope seen head on: CW spin

\section{H. $E P R$}

Many have gone out of their way to provide a physical interpretation to EPR since Einstein's group formulated it in 1936. ${ }^{18}$ The rational thinker is compelled to choose between mystical hidden variables, ${ }^{19}$ a manifold of many worlds, ${ }^{20}$ or particles returning from the future. ${ }^{21}$

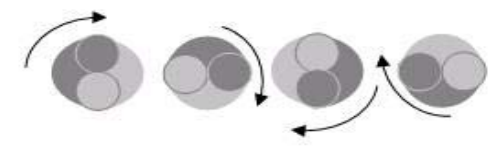

Fig. 14.EPR: CW and CCW spin have simple explanations under the rope hypothesis. At one end the rope torques $\mathrm{CW}$. The other end has no choice but to spin $\mathrm{CCW}$.

Under the rope hypothesis, it is straight forward. Observer A watches the rope turning CW. Observer B at the other end should see the rope spinning $\mathrm{CCW}$ (Fig. 14). If we reverse the spin, A now detects the rope twirling $\mathrm{CCW}$ and $\mathrm{B} \mathrm{CW}$.

\section{Polarization}

One of the most intriguing properties of light is its ability to polarize. Discrete entities such as particles are rarely if ever mentioned in this context. Polarization is unimaginable as anything other than a wave phenomenon.

Fresnel's $19^{\text {th }}$ Century 'picket-fence' analogy is still the 
dominant theory. According to Fresnel, when either the first or second of two polarizers in series is rotated at $90^{\circ}$ to the other, one polarizer filters out the vertical and the second one the horizontal component of the EM transverse wave (Figs. $15 \mathrm{X}$ and $\mathrm{Y}){ }^{22}$ It has been established since then that if a third polarizer oriented at $45^{\circ}$ is inserted between them part of the beam reappears as if by magic (Fig. 15 Z). Many have abandoned hopes of rationalizing this bizarre behavior. Indeed, Paul Dirac declared the matter to be outside the bounds of Science:

"The most that can be predicted is a set of possible results, with a probability of occurrence for each... Questions about what decides whether the photon is to go through or not and how it changes its direction of polarization when it does go through cannot be investigated by experiment and should be regarded as outside the domain of science.".$^{23}$

Under the rope hypothesis, every atom in the source is connected to every atom in the first polarizer and all of them are connected to the second polarizer and to whatever is behind it (Fig. 16 X) . When one of the polarizers is rotated, the ropes connecting the two arrays are no longer parallel with the ropes comprising the incoming beam (Fig. $16 \mathrm{Y}$ ). At the extreme $90^{\circ}$ position of the polarizer the ropes are completely deviated from their refractive angle. Inserting a third polarizer between them at $45^{\circ}$ corrects this situation as ropes between both $\mathrm{A}$ and $\mathrm{C}$ and between $\mathrm{C}$ anf $\mathrm{B}$ approach their refractive angles (Fig. $16 \mathrm{Z}$ ). A second effect - beyond the scope of this paper to deal with it in any detail - comes from the direction of torsion of the individual ropes comprising the beam, a phenomenon that becomes relevant in the creation of circularly polarized light.

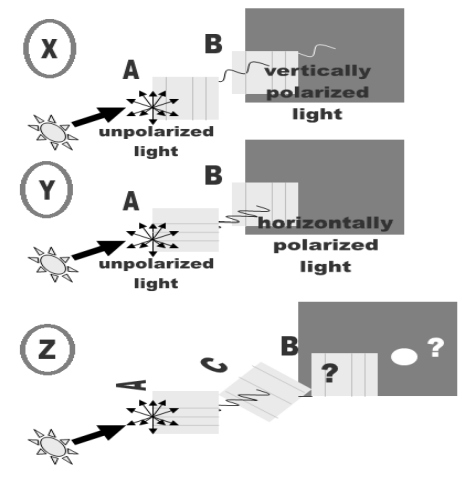

Fig. 15.X: Polarized light. Y: Beam Extinction.

$\mathrm{Z}: 3^{\text {rd }} 45^{\circ}$ polarizer in series - explanation outside the bounds of science.

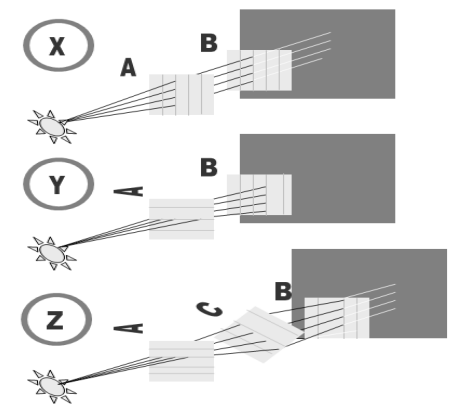

Fig. 16.X: Polarized light. Y: Beam Extinction.

$\mathrm{Z}: 3^{\text {rd }} 45^{\circ}$ polarizer in series under the rope simulation.

\section{J. Gravity}

Perhaps the most important phenomenon that the rope model helps us explain is the Grand Unified Theory (GUT), the Holy Grail of Physics. Researchers have been searching for a mathematical GUT for decades. With particles and waves, that goal is ever more distant. It is not easy, if at all possible, to explain attraction with discrete one-way agents.

The physical interpretations offered to the mathematical solutions certainly strain the imagination. They include scenarios where gravitons and gluons transfer 'negative momentum' to the test object or rely on particles that through contorted mechanisms end up pushing the two objects together. $^{24}$

With the rope hypothesis, we finally have a chance to imagine a straight forward, physical mechanism that can produce attraction seemingly from a distance. If every atom in the Universe is bound to all others, it doesn't stretch the imagination to take the vision to the next step and theorize that as one object approaches another, the EM ropes fan out as a function of decreasing distance and cause the acceleration of one to the other (Fig. 17).

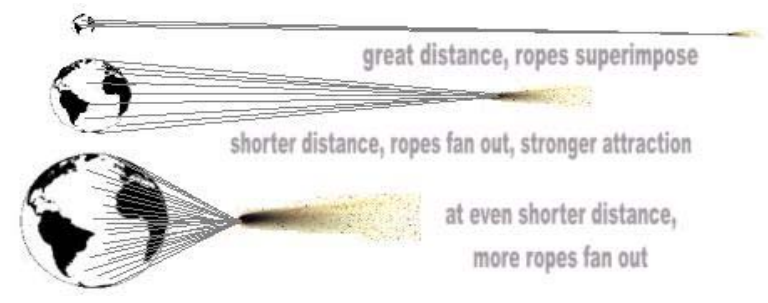

Fig. 17.Action-at-a-distance mediated by EM ropes. At great distances, the ropes superimpose and act as one. At short distances, the ropes fan out and act individually, compelling the comet to accelerate towards Earth.

\section{CONCLUSions}

The issue before the reviewers of this paper is strictly qualitative: Is there a physical medium that underlies light?

If we have already made up our minds that there is not a physical medium, we may be missing the opportunity to gain a deeper understanding of nature. We may never be able to explain phenomena that continue to elude us.

If instead the juror concludes that there is in fact an invisible entity that underlies light, we must simply go through the checklist. Does a rope have frequency, wavelength and amplitude? Does a torsion wave travel practically instantaneously along a rope? Does the rope architecture justify Faraday, Ampere and Maxwell Laws? Is the equation $\mathrm{c}=f * \lambda$ the mathematical description of a rope? And so on.

The rope hypothesis offers not only simplicity and symmetry - features generally yearned by theorists - but as a bonus is also readily amenable to illustration. This makes it a very practical didactic tool.

There is a tendency of conservatism to reject a radical proposal outright because it fails to conform to textbook physics, threatens strongly-held traditional beliefs, or doesn't visibly answer questions about light not covered in the instant paper that are certain to arise, such as how the EM ropes avoid tangling. It is as a result of these potential biases that 
we have limited our analysis to the most obvious properties and behaviors of light that the rope hypothesis is able to justify. The purpose of this paper is not to explain all the known attributes of light, but to present the model in its most rudimentary form in order to introduce the subject to the scientific community. The juror is asked to evaluate the instant proposal objectively and strictly within the claims made herein.

We note, finally, that it is in the spirit of science to afford new theories as much latitude as possible. If the model does not succeed despite all efforts to save it, judge and jury can take pride in their work and rest assured that there was no discretionary abuse when issuing a decision and a verdict.

\section{REFERENCES}

[1] N. Bohr, "The Quantum postulate and the recent development of atomic theory," Nature (Supplement) 121, 1928, 580-590

[2] A. Einstein, "On a Heuristic Viewpoint Concerning the Production and Transformation of Light." Annalen der Physik 17, 1905, pp. 132-148.

[3] R. Baierlein, "Newton to Einstein," Cambridge University Press (1996) p. 170.

[4] C. Fowler, "The solid earth: an introduction to global geophysics" (2nd ed.). Cambridge University Press., 2005.

[5] F. Arago and A. Fresnel, "Sur l'Action que les rayons de lumière polarisés exercent les uns sur les autres," Ann. Chim. et Phys. 10 (1819), 288-305.

[6] M. Faraday, "Experimental researches in electricity" (Bakerian lecture), Philos. Trans. R. Soc. London, 122, 1832, 163- 177.

[7] A. Ampère, "Memoir on the Mathematical Theory of Electrodynamic Phenomena, Uniquely Deduced from Experience" 1827.

[8] J. Maxwell, "A dynamical theory of the electromagnetic field," Phil. Trans. 155, 1865, $459-512$.
[9] Z. Knittl, "The use of the principle of reversibility in deriving known relations for a system of optical thin films," Czech. J. Phys. 7, 1957.

[10] A. Mahan, “A Mathematical Proof of Stokes' Reversibility Principle," J. Opt. Soc. Am. 33, 1943, 621-626.

[11] A. Schuster, "An Introduction to the Theory of Optics," London: Edward Arnold, 1904.

[12] J. Williams, J. Dickey, "Lunar Geophysics, Geodesy, and Dynamics," 13th International Workshop on Laser Ranging, October 7-11, 2002, Washington, D. C.

[13] F. Grimaldi, "Physico mathesis de lumine, coloribus, et iride, aliisque annexis libri duo," Bologna, Italy: Vittorio Bonati, 1665, pages 1-11.

[14] T. Young, Phil. Trans. Roy. Soc. 12, London xcii, 1802, 387.

[15] P.Merli, G. Missiroli, G. Pozzi, "On the statistical aspect of electron interference phenomena," Am. J. Phys. 44, 1976a, 306-307.

[16] A. Tonomura, J. Endo, T. Matsuda, T. Kawasaki, "Demonstration of single-electron buildup of an interference pattern," Am. J. Phys. 57 (2), 1989, 117-120.

[17] M. Born, P. Jordan, „Zur Quantenmechanik, “Zeitschrift für Physik 34, 1925, 858-888. [Eng. Trans. in: B. L. van der Waerden, editor, "Sources of Quantum Mechanics," Dover Publications, 1968.

[18] E. Einstein, B. Podolsky, N. Rosen, Can quantum mechanical description of physical reality be considered complete?," Physic PhysicalReview 47, 1935, 777 777-780.

[19] D. Bohm, Y. Aharonov, "Discussion of Experimental Proof for the Paradox of Einstein, Rosen and Podolski," Physical Review 108: 1957, 1070-1076.

[20] H. Everett, "'Relative State' Formulation of Quantum Mechanics," Reviews of Modern Physics 29, 1957, 454-462.

[21] J. Cramer, “An Overview of the Transactional Interpretation,” Int. J. of Theo. Phys. 27, 227, 1988.

[22] A. Fresnel, "Mechanical considerations on the polarization of the light," Paris (1821).

[23] P. Dirac, "The Principles of Quantum Mechanics", Oxford University Press (1982).

[24] H. Casimir, D. Polder, "The Influence of Retardation on the London-van der Waals Forces," Phys. Rev. 73, 360, 1948. 